\title{
L'histoire de la mécanique statique arabe
}

\author{
BADAOUi El MABSOUT ${ }^{a}$ \\ Institut Jean Le Rond d'Alembert, Université Paris 6, couloir 65-55, pièce 404, 4 place Jussieu, 75005 Paris, France
}

Reçu le 3 mai 2007, accepté le 21 juin 2007

\begin{abstract}
Résumé - Nous avons tracé dans cet article les grandes lignes, connues jusqu'à maintenant, de l'histoire de la mécanique statique écrite en arabe entre le huitième et le quatorzième siècle. Ainsi, nous avons passé en revue les sources grecques de la mécanique statique traduite en arabe. Nous avons résumé, ensuite, les traités de mécanique statique écrits en arabe qui nous sont parvenus. Nous avons évoqué, aussi, les autres traités arabes perdus cités par les biographes ou dont on connait des passages dans des traités qui ont utilisé leurs résultats; il s'agit principalement du traité « Le Livre de la Balance de la Sagesse » écrit par 'Abd al-Raḥmān al-Khāzin̄̄ au treizième siècle. Nous avons enfin cité des traités en mécanique statique écrits en latin, traduits de l'arabe ou inspirés par des traités écrits en arabe dans ce domaine.
\end{abstract}

Mots clés : Histoire / mécanique statique écrite en arabe / équilibre d'une poutre pesante / loi du levier / balance romaine

\begin{abstract}
History of Arab static mechanics. We traced in this paper the broad outline, known until now, of the history of the static mechanics written in Arabic between the eighth and the fourteenth century. Thus, we reviewed the Greek sources of the static mechanics translated into Arabic. We summarized, then, the treaties of static mechanics written in Arabic, which reached us. We evoked, also, the other lost Arab treaties quoted by the biographers or of which we know paragraphs in treaties which used their results; it is mainly about the treaty "the Book of the Balance of Wisdom" written by 'Abd al-Rahmān al-Khāzin̄ at the thirteenth century. We finally quoted of the treaties in static mechanics written in Latin, translated from Arabic or inspired by Arabic treaties written in this field.
\end{abstract}

Key words: History / Arab static mechanics / equilibrium of a heavy beam / law of the lever / roman balance

\section{Introduction}

Nous allons tracer dans cet article les grandes lignes de l'histoire de la mécanique statique écrite en arabe entre le huitième et le treizième siècle. Au début de cette période de l'histoire les traductions des ouvrages scientifiques et philosophiques ont été effectuées à partir des sources indiennes, grecques et syriaques. Cela a permis aux savants arabes d'assimiler tout d'abord les apports des autres civilisations avant de donner leurs propres contributions. Des avancées importantes ont été ainsi obtenues dans tous les domaines scientifiques, y compris en mécanique. Cet apport des savants arabes a été transmis au monde latin à partir du douzième siècle, par des traductions et par des transmissions directes par l'intermédiaire des savants expulsés de l'Espagne au quinzième siècle, ce qui a contribué à l'évolution scientifique qui a eu lieu en Europe occidentale.

\footnotetext{
a Auteur correspondant : bae@ccr.jussieu.fr
}

La mécanique, comme toutes les sciences, s'est élaborée lentement au cours des siècles, grâce à l'apport des différentes civilisations; ce qui a abouti à la théorie dynamique de Newton et à la théorie de la relativité d'Einstein. Malgré la perte dramatique d'une grande partie des manuscrits arabes écrits pendant la période médiévale, nous arrivons à tracer l'histoire de la mécanique statique écrite en arabe, grâce à quelques manuscrits qui nous sont parvenus. Le plus important de ces manuscrits est le « Livre de la Balance de la sagesse » de 'Abd al-Raḥmān al-Khāzin̄̄ écrit au treizième siècle; c'est une véritable encyclopédie qui permet de décrire une bonne partie des avancées théoriques et expérimentales dans ce domaine ${ }^{1}$.

Le mot mécanique est d'origine grecque. Il provient du mot grec $\mu \eta \kappa \alpha \nu \eta^{\prime}$ qui veut dire machine au sens général.

\footnotetext{
1 Nous avons adopté les transcriptions suivantes des sons arabes dont il n'existe pas d'équivalent en lettres latines :

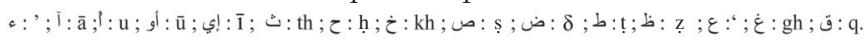


Les grecs connaissaient (Fig. 1) les cinq machines dites simples (voir [1]) : le treuil, le levier, la poulie, le coin et la vis.

La mécanique est devenue par la suite l'art de composer ces machines simples pour inventer des machines plus complexes permettant de déplacer les corps lourds à l'aide de petites forces. Ainsi tout problème qui consistait à trouver des solutions pour mouvoir des corps lourds à l'aide de petites forces, était appelé « problème mécanique ». C'est ce que l'on trouve dans le livre « Les problèmes mécaniques » attribué à Aristote (voir [2]). Le traitement de ces problèmes mécaniques se faisait à l'aide d'outils mathématiques, ce qui a permis de classer la mécanique parmi les sciences mathématiques. Le mathématicien Pappus (troisième siècle et quatrième siècle de l'ère chrétienne) a défini la mécanique (voir [3], livre 8, pp. 809-814) comme la science qui traite les problèmes de statique et d'équilibre ainsi que les problèmes concernant les forces et le mouvement. Par conséquent certains de ces problèmes sont théoriques et dépendent de la géométrie, alors que d'autres sont pratiques et concernent les différentes machines d'ingénieurs (machines hydrauliques, pneumatiques et machines de guerre) ou la construction des bâtiments, la métallurgie et l'équilibre des corps flottants.

La mécanique ancienne s'est scindée ainsi en deux branches :

1. La mécanique au sens ancien qui traite la composition et l'invention des machines. C'est la branche que les savants arabes ont désigné dans un premier temps par « la science des méthodes ingénieuses ». Elle comprend ce que nous désignons aujourd'hui par « l'ingénierie mécanique $»($ voir [4]).

2. La mécanique statique. C'est la branche qui traite les problèmes d'équilibre des corps et des conditions qui entraînent leur repos ou l'arrêt de leur mouvement. Ainsi nous y trouvons un premier groupe de sujets théoriques et pratiques concernant l'équilibre et ses postulats dans lequel on utilise des outils mathématiques. Citons dans ce groupe, à titre d'exemple, « la loi du levier » ou « la loi d'équilibre d'une poutre non pesante », « la loi d'équilibre d'une poutre pesante $»$ et les problèmes des centres de gravité que nous aborderons plus loin. C'est ce qui a été connu sous le nom de « la science des poids et des balances $»$. Un autre groupe de sujets concerne l'équilibre des corps flottants sur l'eau ou immergés dans l'eau, comme les problèmes d'équilibre des bateaux et les problèmes des pourcentages en poids et en volume des métaux constituant un alliage donné. C'est ce que l'on peut désigner par « la mécanique statique hydraulique $»$.

Cependant « la science des méthodes ingénieuses * comprend en général, chez les savants arabes « la science des poids et des balances ». C'est Muhammad ibn Aḥmed al-Khuwārizmī (dixième siècle) qui classifie cette science, dans son livre « Mafātih al-'Ulūm » ou « Les clés des Sciences », parmi les sciences fondamentales comme la philosophie, la logique et l'astronomie. Il dit, en effet, (voir [5], p. 80, l. 16-20) :

«Les sciences mathématiques comprennent quatre branches. L'une de ces branches est l'arithmétique ou la science du nombre et du calcul; la deuxième est la géométrie; la troisième est l'astronomie qui est la science des étoiles; la quatrième est la science de la musique, ou science des mélodies. Quant à « la science des méthodes ingénieuses », elle est indépendante des autres sciences y compris des quatre précédentes ».

Il dit aussi (voir [5], p. 141, l. 18-19) : « L'une de ses branches (il s'agit de « la science des méthodes ingénieuses ») est le déplacement des corps lourds par des forces faibles $»$.

D'après le même auteur la seconde branche concerne (voir [5], p. 143, 1. 2-3) « les méthodes ingénieuses des mouvements de l'eau, de la construction des récipients magiques et la construction, qui dépend de la précédente, des machines qui se meuvent par elles-mêmes. »

\section{Les postulats et les problèmes de la mécanique statique}

Nous allons, dans ce qui suit, passer en revue les postulats connus en mécanique statique, exposer les problèmes de la mécanique statique traités dans les références grecques et arabes ainsi que les lois qui résultent de ces problèmes.

\subsection{Les postulats}

\subsubsection{Premier postulat}

Si deux distances dans un plan sont parcourues par deux mobiles respectifs en deux temps égaux, alors le rapport de l'une des distances à l'autre est égal au rapport de la force du moteur du premier mobile dans son parcours plan à la force du moteur de l'autre mobile.

\subsubsection{Deuxième postulat}

Prenons une poutre rectiligne uniforme et homogène que nous suspendons en son point milieu, alors cette poutre se met en équilibre parallèlement à l'horizon. (C'est pourquoi on peut alors la considérer comme une «poutre non pesante », comme un « segment de droite» ou comme « une droite », en abrégé.)

\subsubsection{Troisième postulat}

Soit une droite divisée en deux parties égales. On suspend, aux deux extrémités de cette droite, deux poids égaux, et on la suspend en son milieu. Alors la droite devient parallèle à l'horizon. 

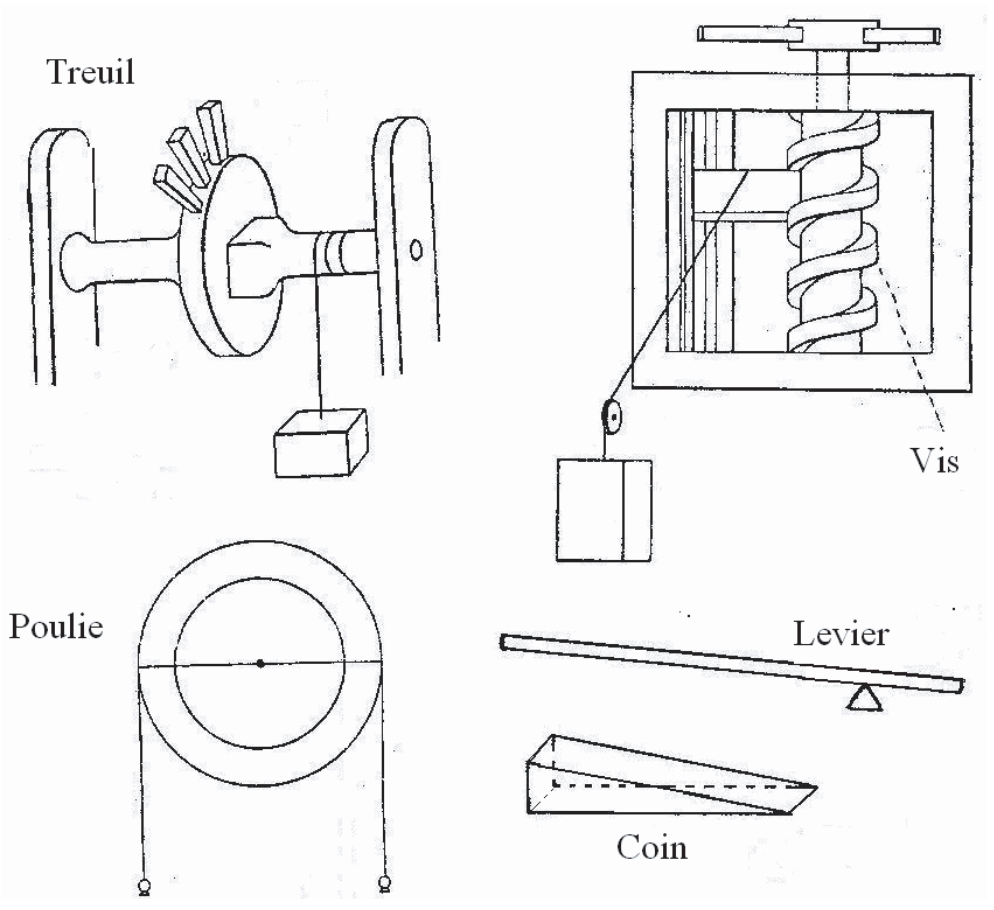

Fig. 1.

\subsubsection{Quatrième postulat}

Prenons une droite suspendue en l'un de ses points et qui devient en équilibre parallèlement à l'horizon lorsque deux poids sont suspendus à ses deux extrémités. Si l'on retire les deux poids des deux extrémités pour les suspendre sur deux perpendiculaires à cette droite menées par ces deux extrémités, alors ces deux poids restent en équilibre. Si les longueurs de ces perpendiculaires sont différentes, l'état d'équilibre de la droite parallèlement à l'horizon n'est pas modifié. De même si les directions de ces perpendiculaires sont différentes, la droite reste parallèle à l'horizon, car la différence entre les longueurs des perpendiculaires ne modifie pas l'attraction des deux extrémités de la droite vers le bas; de même la différence entre les directions ne modifie pas l'attraction des deux extrémités de la droite vers le bas; une telle différence crée plutôt un mouvement de rotation qui ne modifie pas le parallélisme à l'horizon.

\subsubsection{Le cinquième postulat}

Tout corps pesant se dirige vers le centre du monde (la terre) à moins qu'il en soit empêché par un obstacle.

\subsection{Les problèmes de la mécanique statique}

\subsubsection{Le problème de l'équilibre d'une poutre non pesante} et la loi du levier

Prenons une poutre, rectiligne uniforme et homogène, que l'on suspend en son milieu. Cette poutre se met en équilibre parallèlement à l'horizon, d'après le deuxième postulat cité plus haut. On peut d'ailleurs vérifier cela par l'expérience. On obtient ainsi une poutre dite non pesante que l'on peut assimiler à un segment de droite ou à une droite en abrégé. Alors si on suspend un poids $d$ en un point de l'un des deux côtés de la poutre à une distance $f$ du point de suspension $C$ et si l'on suspend de l'autre côté un poids $e$ à une distance $g$ de l'axe, on se demande quelle est la relation entre le rapport des poids $d$ et $e$ et le rapport des distances $f$ et $g$ qui permet d'avoir la poutre en équilibre parallèlement à l'horizon. C'est le problème que nous désignons par « le problème de l'équilibre d'une poutre non pesante $»$. La solution de ce problème donne ce que l'on appelle « la loi du levier » ou « la loi de l'équilibre d'une poutre non pesante $»$. Le levier $(\mu v \chi \lambda$ en grec et mukhl en arabe) est une poutre (ou un bâton) solide de section ronde ou carrée qui est utilisée pour déplacer des objets lourds. Pour cela on creuse un petit trou sous l'objet et on y introduit une extrémité du levier, puis on met une pierre sous le levier à côté du trou; alors si l'on appuie de haut en bas sur l'autre extrémité du levier, l'objet lourd se déplace très facilement; le déplacement de cet objet est d'autant plus facile que la pierre posée sous le levier est plus proche de l'objet.

La loi dite du levier ou « la loi de l'équilibre d'une poutre non pesante $»$ s'énonce comme suit (Fig. 2) :

- si une droite AB est suspendue par l'un de ses points $\mathrm{C}$ et si on suspend à ses deux extrémités $\mathrm{A}$ et $\mathrm{B}$ deux poids respectifs $P_{\mathrm{A}}$ et $P_{\mathrm{B}}$ inversement proportionnels aux longueurs des deux parties $\mathrm{AC}$ et $\mathrm{BC}\left(P_{\mathrm{A}} / P_{\mathrm{B}}=\right.$ $\mathrm{BC} / \mathrm{AC})$, alors la poutre est en équilibre parallèlement à l'horizon. 


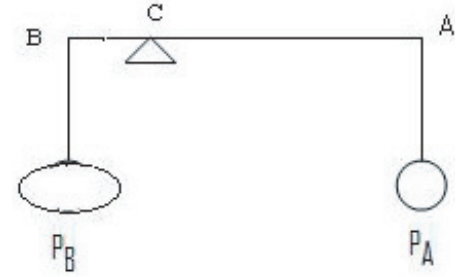

Fig. 2.

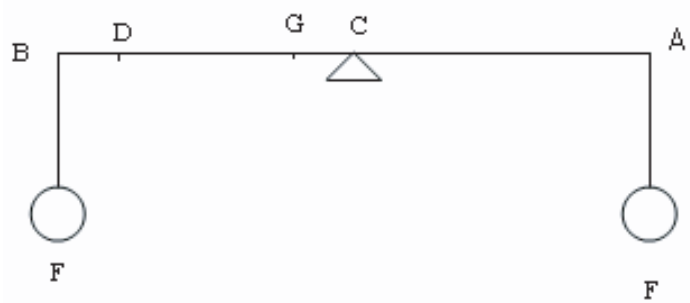

Fig. 3.

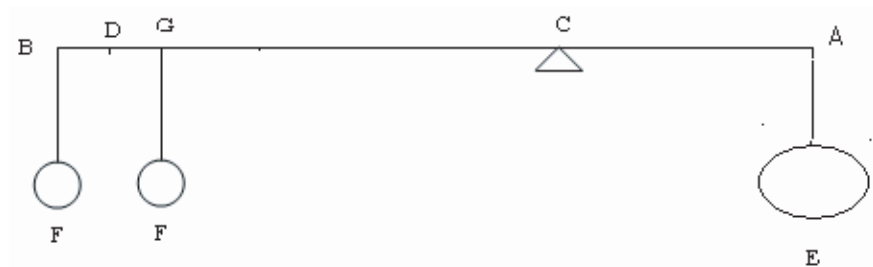

Fig. 4.

\subsubsection{Le problème de distribution des poids}

Prenons une poutre AB (Fig. 3) uniforme et homogène suspendue par son point milieu C. Suspendons à ses deux extrémités deux poids égaux respectifs de grandeur $F$, afin qu'elle soit en équilibre parallèlement à l'horizon. Si on retire le poids suspendu en B pour le suspendre au point $\mathrm{D}$ situé à une distance $d$ de l'extrémité $\mathrm{B}$ où il était suspendu et si l'on suspend un autre poids de grandeur $F$ au point $\mathrm{G}$ situé sur $\mathrm{BC}$ à une distance $d$ de l'axe $\mathrm{C}$, alors la poutre reste en équilibre parallèlement à l'horizon. C'est ce résultat qui s'appelle « la loi de distribution des poids $»$.

\subsubsection{Le problème du remplacement pour deux poids égaux}

Soit une poutre AB sans poids (Fig. 4) suspendue en l'un de ses points C. On suspend à l'une de ses extrémités, soit A, un poids $E$; on suspend de l'autre côté deux poids égaux de grandeur $F$, l'un à l'extrémité $\mathrm{B}$ et l'autre en un point $\mathrm{G}$ qui se trouve entre $\mathrm{B}$ et $\mathrm{C}$; on suppose que ce dispositif (voir Fig. 4) est alors en équilibre. Maintenant si on retire les deux poids suspendus en B et $G$ pour les regrouper et les suspendre au point $\mathrm{D}$, milieu de BG, la poutre gardera l'équilibre parallèlement à l'horizon. C'est ce résultat qui peut être désigné sous le nom de « la loi du remplacement pour deux poids égaux ».

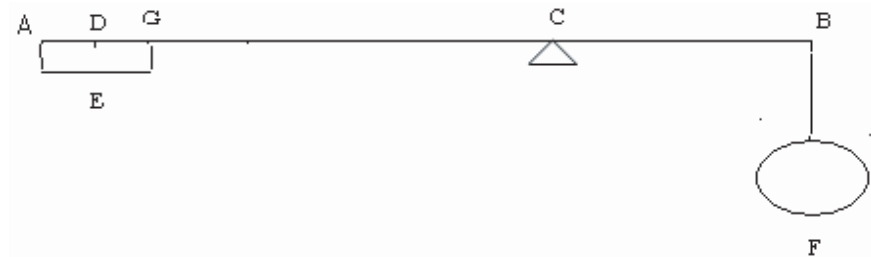

Fig. 5.

\subsubsection{Le problème du remplacement pour un poids uniformément étendu sur un segment d'une poutre non pesante}

Soit une poutre (Fig. 5) AB sans poids, une droite, suspendue en l'un de ses points $\mathrm{C}$; on suppose qu'un poids $E$ est étendu, de façon uniforme et homogène, le long d'un segment $\mathrm{AG}$ situé du côté AC de la poutre, tandis qu'un autre poids $F$ est suspendu au point B pour avoir l'équilibre de la poutre parallèlement à l'horizon. Alors si l'on retire le poids $E$ de sa position, si on le regroupe pour le suspendre au point $G$ milieu de $A D$, la poutre se met en équilibre parallèlement à l'horizon.

C'est ce résultat que l'on peut appeler « loi du remplacement pour un poids uniformément étendu sur un segment d'une poutre non pesante $»$.

\subsubsection{Le problème d'équilibre d'une poutre pesante}

Prenons une poutre rectiligne uniforme et homogène que l'on suspend en l'un de ses points qui n'est pas son milieu. La poutre penche alors vers le bas du côté le plus long. Le problème dit de la poutre pesante consiste à savoir quelle est la grandeur du poids qu'il faut suspendre à l'extrémité de la partie courte de la poutre afin qu'elle soit en équilibre parallèlement à l'horizon. La solution de ce problème nous donne « la loi de l'équilibre de la poutre pesante » que nous énonçons dans ce qui suit telle qu'on la trouve dans les livres anciens :

- Si nous prenons une poutre rectiligne uniforme et homogène suspendue en l'un de ses points différent de son point milieu, et si nous voulons connaître la grandeur du poids qu'il faut suspendre à l'extrémité de la partie courte de la poutre afin que celle-ci s'équilibre parallèlement à l'horizon, alors nous retenons le poids de cette poutre, sa longueur et celle de chacune de ses deux parties, nous prenons la différence entre les longueurs des deux parties, nous la multiplions par le poids de la poutre et nous divisons le résultat obtenu par le double de la longueur de la partie courte de la poutre, ce qui donne la valeur du poids qui, suspendu à l'extrémité de la partie courte, équilibre la poutre parallèlement à l'horizon.

\subsubsection{Les problèmes des centres de gravité}

Il existe pour tout corps un « centre de poids » ou centre de gravité. C'est le point qui permet d'avoir ce corps en équilibre indifférent si on l'y suspend. Ce point, 
selon la forme du corps, peut se trouver à l'intérieur ou à l'extérieur de ce corps. Si le corps est homogène la détermination de son centre de gravité devient un problème géométrique, comme la détermination du centre de gravité d'une droite d'un triangle ou d'un morceau d'une surface plane circulaire d'une sphère ou d'un paraboloïde. D'autres problèmes consistent à trouver le centre de gravité d'un groupe de corps dont les centres de gravité sont connus.

\subsubsection{Les problèmes d'équilibre des corps flottants à la surface de l'eau}

Ces problèmes se résolvent par l'application de la loi d'Archimède qui dit que tout corps, plongé dans un liquide ou flottant à sa surface, subit une force verticale, dirigée vers le haut, dont l'intensité est égale au poids du liquide déplacé. On peut lire par exemple l'énoncé de cette loi dans le livre de 'Abd al-Rahmān al-Khāzinī : « La Balance de la Sagesse » ([6], p. 24, l. 7-9) :

« Tout corps pesant qui se déplace dans un fluide est gêné (handicapé) par lui et réciproquement. C'est pourquoi l'eau gêne le corps pesant dans laquelle on le jette; elle affaiblit sa force et son poids selon son volume. Le poids du corps pesant est alors diminué d'un poids égal au poids en eau de son volume. La gêne (le frottement!) est d'autant plus grande que le volume du corps qui se déplace est grand. »

On arrive ainsi grâce à cette loi à résoudre les problèmes de flottement des bateaux, à étudier leur équilibre et à calculer la longueur de leur partie immergée. De même on parvient à déterminer dans un alliage donné les proportions entre les volumes des métaux qui constituent ce corps. On parvient aussi, en utilisant la loi d'Archimède à fabriquer les densimètres qui permettent de déterminer la masse spécifique par rapport à l'eau.

\section{Les sources grecques de la mécanique statique arabe}

Nous allons passer en revue les sources grecques qui ont été traduites en arabe ou dont nous avons certaines indications sur leur traduction en arabe.

\subsection{Le livre intitulé «Problèmes mécaniques »}

La première de ces sources grecques est le livre intitulé « Problèmes mécaniques ». C'est le plus ancien des livres en mécanique qui nous soient parvenus. Il est attribué à Aristote. Mais il se peut qu'il soit l'œuvre de l'un de ses disciples qui l'a écrit peu de temps après la mort de son maître. Ce livre est perdu en arabe et aussi en latin. On trouve, en effet, dans le livre : « The medieval science of weights $»([7]$ pp. 124-125) ce que l'on peut traduire par :

- « Nous n'avons aucun indice qui prouve que le livre des « Problèmes mécaniques » était connu de la part d'aucun des auteurs latins ni de la part d'aucun des auteurs arabes dont les œuvres dans ce domaine « en mécanique » étaient connues par les latins ».

Mais ce livre n'est pas perdu en grec et il en existe une traduction en anglais parmi les œuvres d'Aristote [2]. Le livre comprend beaucoup d'idées connues d'Aristote comme « la loi du levier $»$ ou « la loi d'équilibre d'une poutre $»$ dont on a parlé plus haut.

L'auteur des «Problèmes mécaniques » dit, entre autres, (voir [7], pp. 4-5) :

« Le bras long de la poutre se meut, sous l'influence du même poids, avec une vitesse plus grande que celle du bras court. Ainsi, la même force cause un mouvement plus grand chaque fois que la distance à l'axe du levier devient plus grande; de même sous l'influence d'un même poids chaque fois que le bras devient plus long, il se meut avec une vitesse plus grande. »

Ainsi l'auteur de ce livre adopte la théorie dynamique d'Aristote en mécanique. Cette théorie se base sur un postulat qui dit que la vitesse d'un mobile est linéairement proportionnelle avec ce que Aristote désigne par « la force du moteur $»$ et qu'elle est inversement proportionnelle à la « résistance du milieu» dans lequel se produit le mouvement. Il se peut que la force du moteur soit identique à ce que l'on appelle « la force du poids », mais il ne faut pas confondre la notion ambiguë de force dont parle Aristote avec la notion moderne de force qui est linéairement proportionnelle à l'accélération et à la masse du corps, selon la théorie dynamique de Newton. Nous signalons aussi que la vitesse dont on parle ici n'est autre que la vitesse moyenne, c'est-à-dire le rapport de la distance parcourue par le mobile au temps nécessaire pour le parcourir.

On trouve dans les documents arabes certains signes qui montrent que le livre des « Problèmes mécaniques » était connu des savants arabes. L'autobiographe al-Qifți (mort en 1248) signale dans son livre « Tarikh al-Hukamā' », ou « histoire des sages », ([8], p. 43) qu'Aristote a écrit un livre en mécanique intitulé « Les problèmes des méthodes ingénieuses $»$.

Ce qui prouve aussi que le livre des « Problèmes mécaniques » était connu de la part des savants arabes est que 'Abd al-Raḥman al-Khāzin̄̄, dans son livre « La balance de la Sagesse », écrit à la quatrième section du premier chapitre du livre V un paragraphe intitulé « sélection des méthodes ingénieuses » qu'il attribue directement à Aristote. Il commence ce paragraphe ([6], p. 99) en disant «Aristote dit...». En fait F. Bancel a démontré ([9], p. 21) que ce paragraphe est le résumé du début du livre des « Problèmes mécaniques », en le comparant avec le texte anglais.

Il convient de signaler aussi que Héron d'Alexandrie [1] a emprunté, dans son livre intitulé « La mécanique » ou «Sur l'élévation des corps lourds » que Qustā ibn Luqā a traduit à la fin du neuvième siècle, du grec en arabe, beaucoup d'idées à Aristote et y a introduit en abrégé des parties importantes du livre attribué à Aristote. C'est ce qu'affirme D. R. Hill dans l'introduction que l'on 
trouve dans l'édition de Carra de Vaux du livre de Héron d'Alexandrie ([1], p. 45) :

« À côté de ces emprunts faits par Héron à la pensée aristotélicienne, on rencontre, dans « les Mécaniques », un chapitre entier qui affecte l'apparence d'un véritable extrait et qui ne tend à rien moins qu'à reproduire, bien que sous une forme très abrégée et avec de sérieuses variantes, « les Mécaniques »d'Aristote. »

Ce qui nous intéresse maintenant c'est d'exposer ce que l'auteur du livre «Les problèmes mécaniques » y a écrit sur la mécanique statique. Il donne une démonstration de « la loi du levier » qui s'appuie sur la théorie de la dynamique d'Aristote. Il prend à cet effet une poutre $\mathrm{AB}$ suspendue en l'un de ses points $\mathrm{C}$ alors qu'un poids $d$ est suspendu au point $\mathrm{A}$ et qu'un poids $e$ est suspendu au point $B$ de manière que la poutre soit en équilibre parallèlement à l'horizon. Il affirme alors que tout point pris sur l'un des deux bras de la poutre décrit un arc de cercle qui est d'autant plus grand que le point est plus éloigné du point de suspension ; alors la vitesse du point est plus grande lorsqu'il est plus éloigné du point de suspension. L'auteur considère que, selon la théorie d'Aristote, chacun des deux poids $d$ et $e$ est le moteur de l'autre. Cela veut dire que si, à partir de la position d'équilibre, on écarte le poids $d$ vers le bas, l'autre poids $e$ le fait remonter vers la position d'équilibre. De même si l'on écarte le poids $e$ vers le bas, le poids $d$ le fait remonter vers la position d'équilibre. Il dit aussi que la « force du moteur $»$ se mesure par le produit du poids du corps mobile par la vitesse de son mouvement. Il affirme enfin que l'équilibre de la poutre parallèlement à l'horizon se produit lorsque les forces des deux moteurs aux extrémités de la poutre sont égales. Cela exige que les poids suspendus aux extrémités de la poutre, soient inversement proportionnels aux deux vitesses des deux extrémités et par conséquent aux distances des deux extrémités de la poutre à l'axe, puisque la vitesse de chaque extrémité est proportionnelle au rayon du cercle qu'elle décrit (voir [7], pp. 29-30).

\subsection{Le livre de Héron d'Alexandrie «La mécanique » ou « Sur l'élévation des corps lourds »}

Héron d'Alexandrie a vécu probablement au premier siècle. Son livre intitulé « La mécanique » est le plus important des livres qui nous sont parvenus en mécanique. Le texte grec de ce livre est perdu, mais il nous est parvenu en arabe sous le titre «Sur l'élévation des corps lourds ». C'est Qustā ibn Luqā qui l'a traduit du grec à la fin du neuvième siècle. Le baron Carra de Vaux a édité le texte arabe et l'a traduit en français en 1893 ; il a été ensuite publié en 1988 avec une introduction de D. R. Hill et des commentaires de A. G. Drachmann.

Les historiens arabes ont cité Héron d'Alexandrie dans leurs livres. Sur ce sujet et sur l'influence des livres de Héron d'Alexandrie dans les traités écrits en arabe, D. R. Hill dit (voir [1], introduction, pp. 16-17) :

- « L'historien égyptien al-Kindī (897-961) rapporte que Héron fut un géomètre-expert, qu'il écrivit sur la géographie et la pneumatique et qu'il fabriqua des horloges et d'autres instruments pour mesurer le temps. Ibn al-Nadīm (envi. 936-995) écrit (voir [10]) que l'on trouve parmi ses ouvrages : un livre sur la résolution des incertitudes d'Euclide, un ouvrage sur l'astrolabe, l'élévation des poids, la puissance de la vapeur, des objets qui se meuvent par eux-mêmes. Ibn al-Qifțī [8] (1172-1248) mentionne ses « Pneumatiques » et son livre sur la résolution des difficultés rencontrées dans l'œuvre d'Euclide. Il est donc presque certain qu'il y a eu des traductions arabes de ses ouvrages, bien que la seule à avoir survécu, à notre connaissance, est celle des «Mécaniques ». La meilleure preuve de l'existence des Pneumatiques en arabe est « le Livre des dispositifs ingénieux » écrit par les Banū Mūsā [11], trois frères qui vécurent à Bagdad au neuvième siècle. Environ 25 sur 100 dispositifs qu'ils décrivent sont tirés directement ou avec des modifications des «Pneumatiques » de Philon (voir [12]) ou de Héron, ou des deux à la fois. Leur travail présente cependant des progrès nets par rapport à celui de leurs prédécesseurs, en particulier dans leur utilisation des contrôles automatiques. L'ouvrage des Banū Mūsa (voir [11]) fut utilisé par des ingénieurs islamiques plus tardifs, y compris par le plus grand d'entre eux, alJazarī (voir [13]) qui acheva son livre des machines en 1206. »

Passons en revue maintenant les sujets, concernant la mécanique statique, qui sont abordés dans le traité [1] «Sur l'élévation des corps lourds ».

Nous trouvons dans la section $24 \mathrm{du}$ premier chapitre de ce livre la définition suivante du centre de gravité : «Le centre de gravité » ou « le centre d'inclinaison » est un point tel que, si on y suspend le poids, alors celui-ci est divisé en deux parties égales « par toute surface plane verticale passant par le centre de gravité ». On y rappelle aussi qu'Archimède distingue entre le point de suspension et le centre d'inclinaison, car le point de suspension est un point donné sur le corps ou en dehors du corps tel que si le corps est suspendu par ce point toutes ses parties deviennent égales, c'est-à-dire, il ne se penche ni s'incline.

Nous trouvons dans la section 32 du livre I de Héron d'Alexandrie, un énoncé de « la loi du levier » :

- « Certains spécialistes des balances ont imaginé que si les poids équilibrent les distances alors les poids sont inversement proportionnels aux distances $»$.

De même, on trouve dans la section 23 sur le même sujet :

- « Archimède a démontré que le rapport du poids au poids est, dans cette situation aussi, comme le rapport de la distance à la distance avec permutation $»$.

On trouve, aussi dans le livre II, les sections numérotées de 35 à 41, concernant la mécanique statique. Héron y détermine tout d'abord le centre de gravité, pour un triangle uniforme et homogène, qui est le point d'intersection des médianes. Il en déduit ensuite le centre de gravité pour un quadrilatère et pour un pentagone. Puis, il détermine le centre de gravité pour trois poids suspendus aux sommets d'un triangle et pour cinq poids suspendus aux sommets d'un pentagone. 


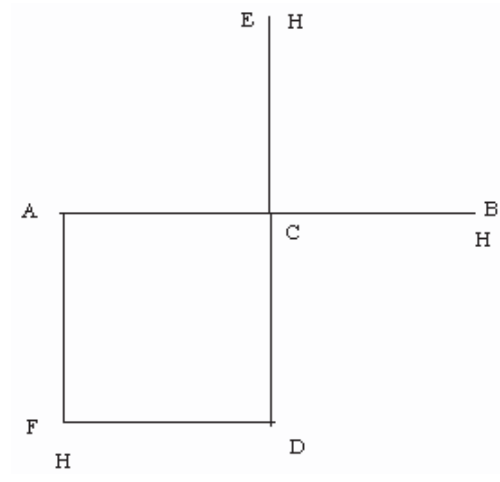

Fig. 6 .

\section{3 «e livre d'Euclide sur la balance »}

Il existe un traité en arabe attribué à Euclide; il nous est parvenu dans un manuscrit sous le titre « Le livre d'Euclide sur la balance ». Ce manuscrit a été édité par F. Woepcke (voir [14]). Il comprend deux postulats, quatre théorèmes et une démonstration de « la loi du levier ». L'auteur de ce manuscrit utilise la théorie statique qui étudie l'équilibre sans aborder les notions de force, de vitesse et de mouvement, mais il utilise la notion de la « force du poids » qui provient de la théorie dynamique d'Aristote dans sa démonstration de la loi du levier.

Le premier théorème que l'on trouve dans ce livre s'énonce ainsi : « Si une surface est suspendue au point $\mathrm{C}$ et si l'on suspend trois poids égaux de grandeur $H$ aux points $\mathrm{B}, \mathrm{E}$ et $\mathrm{F}$ selon la figure 6 :

Alors si l'on suppose que les deux axes ACB et DCE sont orthogonaux et si les distances CE, CD, BC, AC, AF et $\mathrm{DF}$ sont égales, les deux axes sont parallèles à l'horizon.

Le deuxième théorème énonce « la loi de la distribution des poids » que nous avons citée plus haut en parlant des problèmes de la mécanique statique. Dans le quatrième théorème on trouve la démonstration, de « la loi du levier » ou « la loi de l'équilibre de la poutre non pesante », basée sur une méthode statique consistant à appliquer plusieurs fois successives la « loi de distribution des poids ». Cela permet dans un premier temps d'avoir la loi du levier dans le cas particulier où le rapport des deux poids suspendus aux extrémités de la poutre est égal à un nombre entier, avant de généraliser le résultat au cas où ce rapport est égal à un nombre quelconque.

C'est en exposant le troisième théorème que l'auteur du « Traité d'Euclide sur la balance » parle de la notion de « force du poids» pour un corps pesant suspendu à une distance donnée de l'axe. Cette force du poids s'annule lorsque le corps est suspendu sur l'axe; elle grandit de plus en plus si le point de suspension est de plus en plus éloigné de l'axe et si le poids du corps est de plus en plus grand. Cette notion floue de « force du poids » est proche de la notion moderne $\mathrm{du}$ « moment du poids par rapport à l'axe de la poutre $»$ qui est le produit du poids du corps par la distance entre l'axe de la poutre et le point de suspension du corps (voir les détails dans [8], pp. 38-42).

On trouve par ailleurs dans le « Livre de la sagesse * de 'Abd al-Rahman al-Khāzin̄̄ un texte que ce dernier a attribué à Euclide (voir [6], livre I, chapitre trois, pp. 2122 ) intitulé « Sur l'essentiel des problèmes d'Euclide sur la pesanteur et la légèreté, et sur la comparaison des corps les uns par rapport aux autres ».

\subsection{Le livre de Philon de Byzance sur les pneumatiques et les machines hydrauliques}

Philon de Byzance était encore vivant dans le dernier tiers du troisième siècle avant l'ère chrétienne (voir dans [1], l'introduction de D. R. Hill, p. 13).

Il a écrit un traité qui nous est parvenu entier en arabe et il en existe une partie traduite en latin à partir du texte arabe. C'est Carra de Vaux qui a édité le texte arabe et l'a traduit en français en 1903 (voir [12]). Mais ce traité, bien qu'il soit essentiellement écrit de la main de Philon comprend de façon évidente des rajouts arabes dans certains de ses chapitres. Par ailleurs ce livre de Philon a influencé le « Livre des pneumatiques » écrit par Héron d'Alexandrie. C'est que dans ce dernier on peut reconnaître certaines idées prises directement sur le livre de Philon. Mais le livre de Héron n'a pas tout repris sur Philon et l'on y trouve beaucoup d'idées originales.

\subsection{Les traités d'Archimède}

Tous les traités qui nous sont parvenus d'Archimède (mort en l'an 212 avant l'ère chrétienne, à l'âge de 75 ans) sont des traités théoriques (voir [15-17]). On lui attribue l'invention d'un certain nombre de machines comme la vis à eau qui porte son nom, la poulie composée, des machines de guerre et des machines pour soulever les corps lourds. Il existe aussi un traité d'Archimède sur les horloges aquatiques qui nous est parvenu uniquement en arabe : « Le livre sur les horloges de l'eau ». Ce livre comprend des rajouts byzantins et islamiques. Les auteurs arabes l'attribuent à l'unanimité à Archimède ([1], Introduction de D. R. Hill, p. 13).

Nous ne savons pas jusqu'à maintenant si les œuvres d'Archimède en mécanique ont été traduites en arabe ou non (voir [18]). Mais il en existe des traces en arabe dans d'autres traités dont le plus important est « La balance de la sagesse » de 'Abd al-Raḥman al-Khāzin̄̄ (voir [6]). Il existe, en effet, dans ce traité un texte, dans le deuxième chapitre du livre I, pp. 20-21, intitulé « Sur les problèmes d'Archimède sur la pesanteur et la légèreté ».

\subsection{Les traités de Pappus}

Pappus (au quatrième siècle) traite, dans le huitième livre de la collection de ses œuvres en mathématiques, certains problèmes mécaniques concernant les centres de gravité et les forces, ressemblant à celles que traite Héron d'Alexandrie. 'Abdel al-Raḥman al-Khāzin̄̄ donne, dans son livre « La balance de la sagesse » (voir [6], livre I, chapitre 7, pp. 28-33) un texte qu'il attribue à Pappus. Ce texte est intitulé « sur la construction d'un instrument de 
mesure des fluides concernant la pesanteur et la légèreté pour le sage Pappus le byzantin ». Cet instrument de mesure d'après al-Khāzin̄̄ (voir [6], p. 28, l. 16-18), est un dispositif «qui montre, de manière très simple, le rapport du poids de tous les fluides les uns par rapport aux autres s'ils possèdent le même volume et le même degré de légèreté les uns par rapport aux autres ». Autrement dit cet instrument est un densimètre puisqu'il permet de mesurer la masse volumique des fluides ou leur densité par rapport à l'eau.

\subsection{Les traités de Ménélaüs}

Ménélaüs est un astronome mathématicien alexandrin (qui a vécu aux environs de 70-130) qui a introduit la géométrie sphérique en astronomie. Ses œuvres étaient connues en langue arabe, au moins partiellement. Le biographe ibn al-Nadīm (voir [10]) lui cite un livre «sur la connaissance des poids et la distribution des corps », mais ce livre est perdu. Il se peut que 'Abd Al-Rahman alKhāzin̄̄ ait emprunté un texte à ce livre, puisqu'il donne dans son livre « La balance de la sagesse » un texte, qu'il attribue à Ménélaüs (voir [6], livre I, chapitre 4, pp. 2223). Ce texte est intitulé «sur les principaux problèmes de Ménélaüs sur la pesanteur et la légèreté »; on y explique les propriétés des corps plongés dans l'eau selon la loi d'Archimède. Al-Khāzin̄̄ donne un autre texte (voir [6], livre IV, chapitre 4, pp. 86-87) intitulé « sur l'explication de Ménélaüs le sage sur les poids des métaux à l'aide de la balance absolue aérienne et aquatique ». Il explique dans ce texte comment on peut déterminer la proportion en or d'un corps qui est un alliage en or et en argent, en se basant sur la valeur de son poids dans l'air et sur la valeur de son poids dans l'eau.

\section{Les auteurs arabes en mécanique statique}

\subsection{Les Banū Mūsā}

Les Banū Mūsā étaient trois frères : Muhammad, Ahmad et Al-Hassan. Ils étaient des courtisans du Calife al-Ma'mūn (813-833) et de ses successeurs. Ils ont écrit une vingtaine de livres dont un seul livre en mécanique nous est parvenu. C'est le « livre des dispositifs ingénieux» (voir [11]) qui a été écrit à Bagdad vers l'an 850. Il comprend la description d'une centaine de mécanismes utilisés dans la plupart des dispositifs ingénieux, ainsi que des lanternes que l'on remplit et que l'on contrôle automatiquement, un masque protecteur des gaz adapté pour l'utilisation dans les puits pollués et une tenaille mécanique. Les Banū Mūsā ont écrit un livre sur la balance, mais il ne nous est pas parvenu.

\subsection{Thābit ibn Qurra}

Thābit ibn Qurra était l'un des savants les plus éminents à Bagdad au troisième siècle de l'Hégire. Il est né à Harran et partit à Bagdad à la suite d'une convocation de Muhammad ibn Mūsā; il a travaillé avec lui et avec ses deux frères Ahmad et Hassan. Il est resté à Bagdad jusqu'à sa mort en l'an 901. Thābit ibn Qurra a effectué des recherches en mathématiques, en astronomie et en mécanique statique. Nous allons, dans ce qui suit, parler de ses deux traités en mécanique statique : « Kitāb fì al-Qarasțūn » et « Ziāda fì al-Qarasțūn ».

\subsection{1 « Kitāb fĩ al-Qarastūn »}

Le traité « « Kitāb fì al-Qarasțūn » ou « livre sur la balance romaine » est l'un des plus importants livres en mécanique statique qui nous soient parvenus en langue arabe. Il semble que le mot « qarasțūn » est d'origine grecque $(\chi \alpha \rho \iota \sigma \tau \iota \omega \nu)$. Il désigne la balance simple, dite romaine, constituée d'une poutre pesante rectiligne uniforme et homogène que l'on divise en deux parties inégales en l'un de ses points qui sera le point de suspension ou axe de rotation.

Ce livre a été conservé dans deux manuscrits : celui de l'Université Saint Joseph de Beyrouth et celui de India Library Office de Londres. Ces deux manuscrits sont en grande partie identiques, mais chacun d'eux contient une scholie qui ne figure pas dans l'autre. Par ailleurs E. Weidmann a publié en 1911-1912 une traduction en allemand de ce livre (voir [19]) basée sur un troisième manuscrit qui se trouvait à la bibliothèque Staatbibliotek de Berlin, mais ce manuscrit est actuellement perdu.

Nous signalons que « Kitāb fī al-Qarastūn » a été édité et publié en 1976 par K. Jaouiche (voir [20]), mais l'auteur s'est basé uniquement sur le manuscrit de Londres sans tenir compte du manuscrit de Beyrouth, sans compter en plus d'autres défauts, ce qui diminue la valeur de cette édition.

Le livre « Kitāb fì al-Qarasțūn » traite dans sa plus grande partie la résolution du problème de la poutre pesante. Thābit ibn Qurra y a exposé aussi les postulats que nous avons cités plus haut.

Thābit ibn Qurra expose tout d'abord la solution du «problème de la poutre non pesante ». Cette solution n'est autre que « la loi du levier ». Il s'appuie dans sa démonstration de cette loi sur la théorie d'Aristote. Il admet en effet que :

a) chacun des deux poids suspendus aux extrémités de la poutre est le moteur de l'autre;

c) la force du moteur de chacun des deux poids est le produit du poids mobile par la vitesse de son mouvement;

d) l'équilibre a lieu lorsque la force du moteur de l'un des deux poids est égale à celle de l'autre;

e) la vitesse du poids mobile est proportionnelle à la force du moteur.

Par ailleurs, Thābit ibn Qurra dit que si la poutre est pesante, si on la suspend en un point qui la divise en deux parties inégales et si on augmente la grosseur de la partie courte jusqu'à ce que la poutre s'équilibre parallèlement à 
l'horizon, alors la poutre devient équivalente à une poutre non pesante.

Il démontre dans son livre « la loi de remplacement des poids » que nous avons citée plus haut. Il la généralise en prenant un nombre pair quelconque de poids égaux suspendus, sur un segment de l'un des deux bras de la poutre, symétriquement par rapport au milieu de ce segment, l'équilibre de la poutre étant assuré grâce à un poids suspendu à l'extrémité de l'autre bras. Cet équilibre se maintient si on regroupe ces poids pour les suspendre au milieu du segment considéré.

Cela permet à Thābit ibn Qurra de démontrer « la loi du remplacement pour un poids étendu sur un segment d'une poutre non pesante ». Pour cela il raisonne par l'absurde en démontrant que la poutre ne peut pas se pencher ni d'un côté ni de l'autre, donc elle est en équilibre parallèlement à l'horizon.

Ainsi Thābit ibn Qurra arrive au «problème de l'équilibre d'une poutre pesante » qu'il résout en utilisant les résultats précédents; il trouve donc « la loi de l'équilibre de la poutre pesante » qui donne la valeur du poids qu'il faut suspendre à l'extrémité de la partie courte de la poutre afin qu'elle devienne en équilibre parallèlement à l'horizon.

Thābit décrit ensuite comment on construit un « qarastūn » en prenant un plateau avec ses crochets que l'on suspend à l'extrémité de la partie courte de la poutre, de manière que l'équilibre de la poutre soit atteint; la poutre devient alors comme une poutre non pesante parallèle à l'horizon. On peut alors l'utiliser pour peser tout objet que l'on met dans le plateau. À cet effet, on dessine des graduations régulières sur la partie longue de la poutre délimitant des parties égales dont la longueur a un rapport connu rationnel avec la longueur de la partie courte. En déplaçant, sur cette partie graduée, un poids connu dit « rummāna », ou grenade, on arrive à obtenir l'équilibre de la poutre, à une distance connue de l'axe, ce qui permet de déterminer le poids recherché.

\subsubsection{Le livre « Ziyāda fī al-Qarasțūn »}

Le manuscrit de Beyrouth du « Kitāb fī al-Qarasțūn » comprend un autre texte intitulé « Ziāda fì al-Qarasțūn », ou «Appendice sur la balance romaine ». Il comprend quatre théorèmes et deux corollaires. Nous produisons, dans ce qui suit à titre d'exemple l'énoncé du premier théorème :

« Soit une poutre uniforme et homogène divisée en deux parties inégales par le point de suspension. On suspend à l'extrémité de la partie courte un poids dont le rapport au poids de la partie longue est égal au rapport de la moitié de la partie longue à la longueur de la partie courte. On suspend aussi au milieu de la partie longue un poids dont le rapport au poids de la partie courte est égal au rapport de la moitié de la longueur de la partie courte à la moitié de la longueur de la partie longue. Alors la poutre se met en équilibre parallèlement à l'horizon ».
La démonstration de ce théorème utilise « la loi du levier $»$ et « la loi du remplacement pour un poids étendu sur un segment d'une poutre non pesante ».

Les autres théorèmes et les deux corollaires sont inspirés de « la loi d'équilibre d'une poutre pesante », sauf le théorème 3 qui est la réciproque du théorème qui donne « la loi d'équilibre d'une poutre pesante ».

\subsubsection{Les travaux de Thābit ibn Qurra cités dans «La balance de la sagesse »}

Al-Khāzin̄̄ écrit dans le premier chapitre du livre II de son traité (voir [6], pp. 33-38) une section intitulée «Sur la description de la pesanteur et de la légèreté de Thābit ibn Qurra » qui comprend une introduction et cinq sous-sections.

Dans l'introduction, il parle du problème du qarasțūn qui permet à un petit corps de contrebalancer un autre dont le poids est beaucoup plus grand que celui du premier, «c'est ce que l'on n'accepte pas que si on le vérifie. Il donne ensuite les deuxième, troisième et quatrième postulats, avant de donner quatre conditions qui permettent de rendre une poutre en équilibre parallèlement à l'horizon :

- Les deux côtés de la poutre doivent être tous les deux dans l'air ou tous les deux dans l'eau ou dans un même fluide ;

- Les deux côtés doivent être d'une même matière;

- La position de l'axe de la poutre et du crochet doivent être au milieu même de « l'épaisseur de » la poutre afin que la longueur des deux côtés soit la même;

- La poutre doit être rectiligne et non tordue.

Les quatre sous-sections qui suivent l'introduction sont consacrées à l'étude de la position de la poutre si l'une des quatre conditions précédentes n'est pas vérifiée. La cinquième sous-section contient le premier postulat qui se trouve dans « Kitāb fī al-Qarastūun ».

On voit ainsi que Al-Khāzin̄̄ emprunte à Thābit ibn Qurra des fragments de «Kitāb fì al-Qarasṭūn»; il développe les idées qui s'y trouvent en rajoutant des détails et des commentaires.

\subsection{Muḥammad ibn Zakariyya al-Rāzī}

Al-Khāzin̄̄ reproduit dans le troisième chapitre du livre IV de « La balance de la sagesse » (voir [6], pp. 8386) un texte qu'il attribue à Muhammad ibn Zakariyya al-Rāzī (864-923) intitulé «Sur la balance naturelle et son utilisation, par Muhammad ibn Zakariyya al-Rāzī ». Ce texte concerne l'utilisation d'une balance ayant deux plateaux identiques de même poids. L'un des deux plateaux est fixe sur la poutre; on y met l'argent, alors que le deuxième plateau dans lequel on met l'or, est mobile sur la poutre. La balance se met en équilibre si la poutre est suspendue en son milieu et si le plateau mobile est suspendu à l'extrémité de la poutre. On met une quantité d'or de poids connu dans le plateau mobile et on met dans l'autre une quantité d'argent qui rend la balance 
en équilibre; puis on remplit les deux plateaux avec de l'eau, alors le plateau qui contient de l'or se penche vers le bas; pour rétablir l'équilibre on déplace l'anneau de ce plateau sur la poutre dans la direction de l'axe; la position de cet anneau qui donne l'équilibre est repérée par un trait qui correspond à la position de l'or pur. Cela permet, si l'on répète l'opération avec un corps composé d'or et d'argent, d'obtenir une position de l'anneau du plateau mobile située entre la position précédente et l'extrémité de la poutre. On peut alors en déduire la proportion entre l'or et l'argent contenus dans le corps considéré. Al-Rāzì explique ainsi comment on peut effectuer la graduation complète et détaillée de cette partie de la poutre.

\subsection{Abū Sahl al-Qūhī}

Abū Sahl al-Qūh̄̄ (aux environs de 940-1000) fut l'un des savants les plus actifs dans le domaine des mathématiques et de l'astronomie dans la deuxième moitié du dixième siècle. Cependant, la plupart de ses traités sont perdus. Il a écrit un livre sur les centres de gravité de certains corps plans ou à trois dimensions, mais ce livre est maintenant perdu et il n'en reste que quelques passages que nous trouvons dans les lettres qu'il a échangées avec un autre savant qui est Abū Ishạaq al-Șābi'. Ce dernier dit dans une lettre à Abū Sahl al-Qūhī (voir [21], p. 38, 1. 20-22 ; p. 39, l. 13-14) : « Je lui rappelle ainsi, qu'il m'avait promis, de son propre gré, d'achever son traité Sur les centres de gravité et de m'en dédier une copie $»$.

Al-Qūhī dit, aussi, dans sa quatrième lettre à al-Ṣābi', en parlant de « la loi du levier » ou de « la loi de l'équilibre de la poutre non pesante $»($ voir $[21]$, p. 84, l. 19 ; p. 86, l. 4 ; p. $85,1.15 ;$ p. $87,1.3)$ :

« J'en jure, par ma vie : le rapport d'un poids à un poids est égal à l'inverse du rapport de la distance à la distance; c'était la prémisse des anciens et c'était pour eux comme l'un des axiomes ainsi que pour ceux qui traitaient de la science des centres de gravité, comme Archimède, Euclide et d'autres mathématiciens, jusqu'à Thābit ibn Qurra et jusqu'à notre temps, et ils n'en ont douté. Nous ne savons pas si sa vérité venait pour eux à partir de l'expérience, et tirée de la sensation, comme l'avait pensé Abū Sa'd al-'Alā' ibn Sahl, ou si elle était l'objet d'une démonstration qui s'est effacée avec le temps, comme d'autres l'avaient pensé. Comment une prémisse qui répond à cette description, qui a eu une telle importance à leurs yeux et qui, à présent, fait l'objet d'une démonstration, pourrait-elle être corrompue par l'expérience?»

Cela montre qu'al-Qūhī n'était pas au courant des démonstrations de cette loi faites par les anciens. Mais il affirme que la démonstration a été effectuée. En fait c'est lui-même qui a réalisé la démonstration; c'est ce qu'il dit à un autre endroit de la même lettre (voir [21], p. 90, l. 14-22; p. 91, l. 10-15) :

« S'il en est ainsi, alors il est vrai que cette prémisse, utilisée par les anciens pour les centres de gravité, n'a besoin ni de condition ni de discussion selon la position, parce que pour deux poids quelconques, quelle que soit leur position, le rapport d'un poids à l'autre est l'inverse du rapport de la distance à la distance entre les trois centres de gravité - je veux dire le centre de gravité de l'ensemble et le centre de gravité de chacun d'eux -, et bien que cette prémisse soit affranchie de toute condition et de toute discussion, elle ne l'est pas d'un peu d'explication; or je l'ai fait dans les centres de gravité et je l'ai démontrée ».

Al-Qūhī dit dans sa deuxième lettre (voir [21], p. 43, 1. 13-15; p. 45, 1. 1-8) qu'il a trouvé, par une preuve géométrique, les centres de gravité de six figures et que ces centres sont disposés dans un ordre étonnant; l'un de ces centres de gravité est celui d'un demi-cercle.

Il affirme dans l'introduction d'un autre traité sur la détermination du volume d'un paraboloïde (voir [22], pp. 835-883) qu'il a arrêté ses recherches sur les centres de gravité après avoir déterminé les centres de gravité d'un certain nombre de figures géométriques dont un secteur de sphère et un ellipsoïde, afin d'étudier le volume d'un paraboloïde dont il a besoin pour déterminer le centre de gravité du paraboloïde.

Par ailleurs al-Khāzin̄̄ a reproduit dans son traité «La balance de la sagesse », certains passages écrits par al-Qūh̄̄, mais ils sont mélangés avec d'autres passages écrits par ibn al-Haytham. C'est le premier chapitre du livre I (voir [6], pp. 15-20) qui s'intitule «Sur les problèmes principaux des centres de gravité d'Abū Sahl al-Qūhī et d'ibn al-Haytham ». Ce chapitre comprend 9 sections. Il semble qu'al-Khāzin̄̄ y a résumé ce qu'il a trouvé utile dans les traités d'al-Qūhī et d'ibn al-Haytham, sans citer les noms de ces traités ni parler en détail de leur contenu et sans distinguer entre ce qui est pris à l'un ou l'autre des deux auteurs. Mais, F. Bancel (voir [23]) a prouvé que les cinq premières sections sont inspirées des écrits d'ibn al-Haytham, alors que les quatre autres sont inspirées par les écrits d'al-Qūhī. Citons donc dans ce qui suit les idées principales que l'on trouve dans ces quatre sections :

- Tout corps pesant qui se meut vers le centre du monde ne le dépasse pas et s'il y parvient son mouvement prend fin. Si son mouvement prend fin l'inclinaison de toutes ses parties vers le centre du monde est la même. Si son mouvement prend fin, sa position par rapport au centre du monde ne change pas.

- Si deux corps sont liés par un lien qui maintient la position de l'un par rapport à l'autre, leur ensemble possède un centre de gravité qui est un point unique qui se trouve sur la droite joignant les centres de gravité des deux corps qui le composent.

- Le centre de gravité de tout corps, dont les surfaces « de ses bords » sont parallèles et dont les parties sont semblables (autrement dit s'il est homogène), est son propre centre « géométrique », je veux dire le point où se croisent ses diamètres. Pour deux corps liés, le rapport du poids de l'un au poids de l'autre, est comme l'inverse du rapport des deux parties, de la droite sur laquelle se trouvent les trois centres de gravité, 
joignant le centre de gravité de l'ensemble à chacun des deux centres de gravité.

- Si deux corps sont en équilibre en un point donné « d'une droite qui les joint », alors le rapport du poids de l'un au poids de l'autre est comme l'inverse du rapport des deux parties de cette droite qui passe par ce point et par les deux centres de gravité de ces deux corps. Si deux corps pesants sont égaux en force, en volume et en forme mais à des distances différentes du centre du monde, alors le plus éloigné du centre du monde est le plus lourd.

\subsection{Abū Sa'd al-‘Alā' ibn Sahl}

Abū Sa'd al-'Alā' ibn Sahl a vécu pendant la même période où Abū Sahl al-Qūhī a vécu (aux environs de 940-1000). Al Qūhī a cité, comme nous l'avons signalé plus haut, dans sa quatrième lettre à al-Șābi', l'avis de Abū Sa'd al-'Alā' ibn Sahl disant que l'exactitude de « la loi du levier $»$ ou de « la loi de l'équilibre de la poutre non pesante » a été vérifiée par l'expérience et par la sensation. Cela montre qu'Abū Sa'd al-'Alā' ibn Sahl s'est intéressé à la mécanique statique et qu'il a rédigé des traités sur ces sujets. Cependant ses traités dans ce domaine ne sont pas connus.

\subsection{Abū 'Abd Allah al-Khawārizmī}

Le livre «Mafātih al-'Ulūm » ou « Les clés des sciences » (voir [5]) écrit par Abū 'Abd Allah Muhammad ibn Aḥmad ibn Yūssef al-Khawārizmī (dixième siècle) est le plus ancien des sources arabes qui parlent des machines simples. Il a été traduit en Latin. Abū 'Abd Allah al-Khawārizm̄̄ y a écrit dans le premier chapitre du livre II (voir [5], pp. 141-142) une description des machines qui permettent de déplacer les corps lourds à l'aide d'une force faible; ce sont les machines signalées par Héron d'Alexandrie dans son livre «Sur l'élévation des corps lourds ». Nous trouvons aussi, dans la deuxième section du deuxième chapitre (voir [5], pp. 143-145) un paragraphe intitulé « Sur les méthodes ingénieuses concernant les mouvements de l'eau et la construction des récipients magiques et tout ce qui en dépend sur la construction des machines qui se meuvent par elles-mêmes ».

\subsection{Al-Hassan ibn al-Haytham}

Al-Hassan ibn al-Haytham était l'un des plus grands mathématiciens de son époque (il était encore vivant en l'an 1040). Il a écrit, selon les biographes, plus de 90 traités en mathématiques, en astronomie, en optique et en mécanique statique. Cependant une bonne partie de ces traités a été perdue, y compris ses deux livres en mécanique statique :

- «Sur les centres de gravité », traité cité par ibn Abī Ușaybi'a dans son livre (voir [24]) : 'Uyūn al-anbā' fì Tabaqāt al-Ațibbā' ou « les meilleures informations sur les catégories de médecins »). Il a été cité aussi par al-Khāzin̄̄ (voir [6], p. 16) et par al-Qalqashand̄̄ (voir [25], vol. 1, p. 476).

- « $\mathrm{F}_{\overline{1}}$ al-Qarastūn » ou « Sur la balance romaine », cité par ibn Ab̄̄ Ușaybi'a (voir [24]), dans son livre signalé ci-dessus, parmi les titres des traités d'ibn al-Haytham.

Dans son livre « La balance de la sagesse », 'Abd al-Raḥmān al-Khāzin̄̄ reproduit dans le premier chapitre du livre I (voir [6], pp. 15-20) un texte intitulé « Les principaux problèmes des centres de gravité d'Abū Sahl al-Qūhī et d'ibn al-Haytham ». Ce chapitre comprend neuf sections dont les cinq premières sont inspirées des écrits d'ibn al-Haytham (voir ce que nous avons dit plus haut sur al-Qūhī).

Il est possible de résumer les idées principales contenues dans ces sections comme suit :

- Le poids est la force qui fait mouvoir le corps pesant vers le centre du monde à moins qu'il y ait un obstacle qui l'en empêche.

- Les corps pesants ont des forces diverses : certains ont des grandes forces; ce sont les corps denses; d'autres ont des petites forces; ce sont les corps légers.

- Si un corps «solide» lourd se déplace dans des corps fluides, son mouvement dépend de leur fluidité; son mouvement est d'autant plus rapide que le corps « dans lequel il se déplace » est plus fluide.

- Les corps ayant des poids égaux sont ceux qui, en se mouvant dans un même corps fluide à partir d'un même point, ont des mouvements égaux, je veux dire qu'ils traversent, en des temps égaux, des distances égales.

- Deux corps sont en équilibre en un point donné si, en les rajoutant à un corps pesant ayant ce point comme centre de gravité, leurs deux centres de gravité se situent de part et d'autre de ce point sur une droite passant par ce point et si la position de ce corps ne change pas, alors que ce point devient le centre de gravité de l'ensemble.

\subsection{Ibn Sīnā (Avicenne)}

Ibn Sīnā ou Avicenne (980-1037) a écrit de nombreux traités encyclopédiques en philosophie, médecine, musique et différentes sciences y compris en mécanique. Son traité «Mi'yār al-'aql » ou « la norme de la raison »se réfère, selon Rozenskaya (voir [26]) au livre « Problèmes mécaniques » d'Aristote et au livre « Sur l'élévation des corps lourds » d'Héron d'Alexandrie (voir [1]). Ce traité comprend deux parties; dans la première ibn Sīnā décrit les cinq machines simples (le treuil, le levier, la poulie, le coin et la vis) selon Héron d'Alexandrie. Dans la seconde partie, il décrit différentes compositions de ces machines et donne beaucoup de détails concernant toutes ces machines composées, dépassant ainsi le travail de Héron d'Alexandrie. 


\subsection{Abū al-Rayḥān al-Bīrūnī}

Abd al-Raḥmān al-Khāzin̄̄ reproduit dans « La balance de la sagesse » (voir [6], livre III, première section) un texte qu'il attribue à Abū al-Rayhāan al-B̄̄rūn̄̄ (9731048) intitulé « Sur les rapports entre les métaux et entre les pierres précieuses ». Il précise, juste après le titre, que c'est une sélection du Livre d'al-Bīrūn̄̄ « Traité sur les rapports entre les métaux et les pierres précieuses en volume $»($ voir $[27])$.

Al-Bīrūn̄̄ parle dans ce texte de la détermination des composants d'un alliage sans séparer ces composants les uns des autres et il dit (voir [6], pp. 55-57) :

« Ce problème a été étudié par les grecs, mais nous n'avons eu connaissance que des travaux de Ménélaüs sur ce sujet. Quant aux travaux modernes, il existe un traité sur ce sujet pour chacun des savants : Sanad ibn 'Alī, Yūḥannā ibn Yūsef, Aḥmad ibn al-Faḍl al-Bukhārī et Muḥammad ibn Zakariyyā al-Rāz̄i. »

Puis il entre dans beaucoup de détails techniques répartis en six sections sur « les rapports entre les métaux fondus et leurs poids par l'observation et l'expérience ».

Par ailleurs, al-Khāzinī donne dans le dixième chapitre (comprenant huit sections) du livre VI un texte dans lequel il expose beaucoup de détails sur les différentes sortes de pierres précieuses et sur leur valeur, en disant qu'il a emprunté ce texte d'un autre traité d'al-Bīrūn̄̄ (voir [28]) intitulé « Le livre de l'ensemble des pierres précieuses ».

\subsection{Al-Muzaffar al-Asfizārī}

Al-Muzaffar ibn Ismā'îl al-Asfizārī est un savant mathématicien mort en 1087. Il a écrit deux traités « Introduction sur les mesures » et « Résumé des éléments d'Euclide ». C'est al-Khāzin̄̄ qui a donné, dans le deuxième chapitre du livre II de « La balance de la sagesse » (voir [6], pp. 38-54), un texte, en quatre sections, qu'il attribue à al-Asfizārī, intitulé « Sur les centres de gravité et la construction de la balance romaine d'al-Muzaffar al-Asfizārī ».

La première section s'intitule « Sur la démonstration des préliminaires des centres de gravité ». Al-Khāzinī y rappelle, en suivant al-Asfizārī, le cinquième postulat cité plus haut, puis il parle du centre de gravité de deux corps regroupés, l'un se fixant à l'autre, en disant que le rapport, de la distance de ce centre à chacun des deux centres de gravité des deux corps, est égal à l'inverse du rapport des poids des deux corps. Puis, il donne deux exemples de deux corps sphériques de même matière mis dans un récipient sphérique ou suspendus dans l'air à un même crochet à la même distance. Il discute alors la position du centre de gravité de l'ensemble des deux corps par rapport aux positions des centres des deux corps sphériques, en fonction du rapport de leurs poids.

La deuxième section du texte attribué à al-Asfizārī (voir [6], pp. 41-46) est intitulée «Sur les lemmes de l'équilibre de la poutre parallèlement à l'horizon ». On y trouve cinq paragraphes comprenant un rappel du cinquième postulat et de « la loi d'équilibre d'une poutre pesante ». Al-Asfizārī présente dans les trois premiers paragraphes un exemple d'une poutre où la partie courte est le cinquième de la partie longue; il démontre alors pour cet exemple, en utilisant « la loi de distribution des poids », la loi de l'équilibre, à savoir que le poids suspendu à l'extrémité de la partie courte est égal à cinq fois le poids suspendu à l'extrémité de la partie longue. Il démontre ensuite la réciproque de « la loi du levier », à savoir, si la poutre est en équilibre parallèlement à l'horizon alors le rapport des deux poids suspendus aux extrémités de la poutre non pesante est égal à l'inverse du rapport des distances des deux extrémités à l'axe. Il démontre cela par l'absurde en utilisant la notion de « la force du poids » suspendu à l'extrémité de la poutre.

Le quatrième paragraphe de la deuxième section, intitulé «Sur le poids équilibrant », commence par l'exposé de « la loi du remplacement de deux poids égaux ». Puis il définit « le poids équilibrant» (voir [6], p. 45) comme étant la grandeur du poids qu'il faut suspendre à l'extrémité de la partie courte de la poutre pesante afin que celle-ci s'équilibre parallèlement à l'horizon. Al-Khāzin̄̄ dit que cette grandeur est déterminée selon « la loi de l'équilibre de la poutre pesante $»$ ou (voir [6], p. 45, 1. 23-24) « selon l'observation et l'expérience ».

Le cinquième paragraphe de la deuxième section ([6], p. 46) est intitulé « Sur le soulèvement du javelot par son extrémité et sur la force nécessaire au poignet de son porteur et sa justification ». Ici al-Asfizārī (ou al-Khāzin̄̄) compare le javelot à la poutre d'une balance, le poignet de la personne qui le porte au crochet ou au point de suspension; ce poignet est aussi comme un poids dans un plateau; la partie longue du javelot est équivalente à son poids suspendu en son milieu.

La troisième section, du texte attribué à al-Asfizārī, est consacrée à la construction de la balance romaine, à la graduation de la partie longue de la poutre et à la pesée. Il dit « il faut que le constructeur de la balance romaine (ou « al-qarastūun ») prenne une poutre, d'une matière solide pouvant supporter les poids à soulever, d'une forme qui facilite le passage d'al-rummāna (poids connu que l'on déplace à l'aide d'un anneau sur la partie longue de la poutre); il faut que la poutre soit uniforme de même épaisseur et homogène. Le premier paragraphe montre comment on divise la poutre en deux parties inégales en un point qui est le point de suspension. L'extrémité de la partie courte s'appelle «al-'aqrab» (ou «scorpion» symbolisant le crochet qui se trouve à cette extrémité), alors que l'extrémité de la partie longue s'appelle le «sinkh» ou bord. On divise la poutre, à partir d'《al-'aqrab» en parties égales correspondant à une ouverture donnée du compas et l'on pratique des rayures correspondant à ces divisions jusqu'au « sinkh » de la poutre, de manière que la distance entre deux rayures consécutives sur la partie longue de la poutre soit une fonction rationnelle connue de l'ouverture du compas. Le poids d'al-rummāna est pris égal au poids de l'une des parties égales situées entre al-'aqrab et le point de suspension, délimitée entre deux graduations consécutives. Le deuxième paragraphe consiste à prendre al-rummāna autrement, en fixant tout 
d'abord son poids arbitrairement, à condition de diviser la poutre entre al-'aqrab et le point de suspension en parties égales de manière que le poids d'une partie délimitée par deux rayures consécutives soit égal au poids d'al-rummāna. Au troisième paragraphe, il demande au constructeur de déterminer « le poids équilibrant » qui est le poids à suspendre à « al-'aqrab » pour avoir la poutre en équilibre parallèlement à l'horizon. Puis, il demande de poser les chaînes de suspension du plateau, le plateau et « al-'aqrab », à condition que la totalité de leurs poids soit inférieure au «poids équilibrant », de mettre dans le plateau le complément du « poids équilibrant» pour réaliser l'équilibre. On procède ensuite à la graduation en détail selon le nombre d'unités de poids. Dans les deux paragraphes suivants, il poursuit la réalisation concrète de la graduation, selon les différents types de balances à réaliser. Au sixième paragraphe, il explique comment on pèse avec cette balance : on met l'objet à peser dans le plateau et on accroche al-rummāna sur la partie longue de la poutre et on la déplace jusqu'à ce que l'équilibre soit atteint; le poids sera alors connu par simple lecture des chiffres correspondant au trait où se trouve al-rummāna.

Al-Asfizārī explique enfin à la quatrième section, sous le titre « Sur la transformation de la balance romaine d'un type de pesée à un autre », comment on peut peser avec la balance des poids non chiffrés sur la partie graduée; cela peut se faire en modifiant le complément du « poids équilibrant » ou le poids d'al-rummāna ou en changeant la place d'« al-'aqrab» ou en rajoutant un poids connu à «al-rummāna ».

Al-Khāzin̄̄ parle d'autres travaux d'al-Asfizārī dans le premier chapitre ([6], pp. 93-102), du livre V de son traité, consacré à « la construction de la balance romaine, son montage, sa vérification et son explication » et intitulé «sur la construction de ses éléments selon les indications d'al-Muzaffar al-Asfizārī ». Ce chapitre comprend sept sections.

Les trois premières sections sont consacrées à la façon de choisir la poutre, sa languette (qui a la forme d'une tête aiguë de javelot, deux bords aiguisés comme ceux d'une épée), une base arrondie fixée à angle droit au milieu de la poutre, le cadre métallique de la languette formé d'une barre horizontale contenant une fente pour accrocher la balance, et de deux côtés situés de part et d'autre de la languette.

La quatrième section intitulée «sur la connaissance complète et absolue de l'ajustement de l'axe, de la fente et du poids $»$. On y trouve une étude intéressante sur la stabilité de l'équilibre de la poutre comprenant trois cas. Il dit (voir [6], pp. 96-97) :

« Si la poutre est de forme cylindrique séparée de la languette, l'axe peut se trouver par rapport à elle dans trois cas différents :

- l'axe de l'équilibre « indifférent » : c'est le cas où l'axe passe par le centre de gravité de la poutre en son milieu réel, perpendiculairement à la longueur de la poutre. Dans ce cas la poutre est facile à tourner par le peseur, elle s'arrête là où il la laisse dans sa rotation.
- l'axe du basculement : c'est le cas où l'axe se trouve entre le centre du monde et le centre de gravité de la poutre (c'est-à-dire que l'axe se trouve sous le centre de gravité de la poutre). Alors si l'on fait mouvoir la poutre elle bascule évidemment car la flèche qui sort du centre du monde la divise en deux parties inégales dont l'une la fait pencher vers le bas, alors elle bascule.

- l'axe de la stabilité : c'est le cas où l'axe se trouve audessus du centre de gravité de la poutre; alors si elle se meut, la flèche qui sort du centre du monde vers son centre de gravité la divise en deux parties inégales, elle balance par conséquent avant de s'arrêter en équilibre permanent parallèlement à l'horizon là où la flèche la divise en deux parties égales $»$.

Al-Asfizārī traite de nouveau ces trois cas lorsque la languette avec son cadre est fixée au milieu de la poutre et lorsqu'on ajoute aussi les trois plateaux ou les cinq plateaux.

La cinquième sous-section intitulée « sur le choix des plateaux » est destinée à la description et à la fabrication des cinq plateaux de « la balance de la sagesse », à savoir, les deux plateaux aériens, « le plateau aquatique » (à usage dans l'eau) que l'on accroche à un anneau sous l'un de ces deux plateaux, le plateau ailé et le plateau mobile qui sont de même poids.

Les sixième et septième sections sont consacrées à la mise des anneaux pour accrocher « le plateau aquatique » et le seau d'eau dans lequel plonge ce dernier plateau.

On constate ainsi que le travail d'al-Asfizārī, comme nous informe al-Khāzin̄̄, est destiné aux applications dans sa plus grande partie. Il est destiné à donner à l'artisan tous les détails nécessaires pour la fabrication correcte de la balance.

\subsection{1 'Umar al-Khayyām}

Al-Khāzin̄̄ donne dans le cinquième chapitre du livre IV (voir [6], pp. 87-92) un texte en quatre sections, qu'il attribue à 'Umar al-Khayyām (1040-1131), intitulé « Sur la balance absolue de l'eau de l'imam 'Umar al-Khayyām, son utilisation et les preuves le concernant lorsque les deux plateaux ou l'un d'eux sont dans l'eau ». Il s'agit en fait de connaître la quantité d'or et la quantité d'argent contenues dans un corps composé d'or et d'argent. On pèse l'or pur et l'argent pur dans l'air dans une balance ayant une poutre uniforme, puis on met l'or dans l'un des deux plateaux qui se trouve dans l'eau, et dans l'autre on retient la valeur du poids qui établit l'équilibre, ce qui donne, pour l'or, le rapport du « poids aérien $»$ au « poids aquatique ». Par la même méthode on détermine, pour l'argent, le rapport $\mathrm{du}$ « poids aérien » au « poids aquatique $»$. Puis, on détermine, pour le corps composé, le rapport du « poids aérien » au «poids aquatique $»$. La comparaison de ce rapport avec les deux rapports précédents permet alors de déterminer la proportion en or et en argent dans le corps composé. Il procède ensuite à la justification de ce procédé et en donne un exemple et une démonstration algébrique. 


\subsection{2 'Abd al-Raḥmān al-Khāzinī}

'Abd al-Rahmān al-Khāzinī a écrit son traité « Kitāb Mīzān al-Ḥkma » ou « Le Livre de la Balance de la Sagesse » (voir [6]) en l'an 515 de l'Hégire (selon ce qui est écrit dans la page de garde du livre édité à Haydarābād) correspondant à l'an 1121 de l'ère chrétienne. C'est un livre destiné aux artisans constructeurs des balances. En effet, il s'intéresse surtout à l'énoncé des résultats théoriques concernant les différents sujets de la mécanique statique donnés par les différents auteurs, sans en donner que rarement les preuves, car les artisans n'en avaient pas besoin. Il abonde dans l'explication de la construction d'un bon nombre de balances, connues en son temps, dont « la balance de la sagesse » qui comprend cinq plateaux et qui permet de peser les poids avec précision et de déterminer la proportion de chaque métal dans un alliage ou un corps composé donné. Al-Khāzin̄̄ énumère les utilités de « la balance de la sagesse », dans le premier chapitre du livre I ([5], pp. 4-5), en affirmant au début de ses propos que la balance de la sagesse « a été inventée par les pensées et complétée par l'expérience et la vérification ».

Le traité «Le livre de la balance de la sagesse » constitue à lui seul une encyclopédie complète contenant de nombreux passages pris dans les différents traités en mécanique statique qui existaient en arabe à l'époque d'al-Khāzinī. Nous avons signalé plus haut ce que al-Khāzin̄̄ a cité sur les écrits en mécanique statique des auteurs grecs, à savoir, Aristote, Archimède, Ménélaüs et Pappus, ainsi que sur les écrits en arabe d'autres auteurs sur ce sujet. Les passages, pris sur des traités de mécanique statique écrits en arabe, sont d'autant plus précieux qu'une bonne partie de ces traités a été perdue.

\subsection{D'autres auteurs}

Al-Khāzin̄̄ cite dans son livre, selon l'ordre chronologique, les noms de quelques autres auteurs qui ont écrit en mécanique statique sur « la position de la balance aquatique $»$ (voir [6], p. 7, 1. 14-21; p. 8, 1. 1-14) dont des auteurs du temps du calife al-Ma'mūn : Sanad ibn 'Alī, Yūhannāibn Yūsef et Aḥmad al-Massah (nous ne sommes pas au courant des traités de ces trois auteurs) et dont un auteur du temps de l'état daylamī : ibn al-'Amīd (nous ne connaissons pas de traité pour cet auteur).

\section{Les traités arabes en mécanique statique transmis en latin}

\section{1 «iber Karastonis »}

Le traité « Kitāb fī al-Qarasțūn » de Thābit ibn Qurra était l'un des traités les plus importants écrits sur les balances pendant la période médiévale. Il a été traduit en latin par Gérard de Crémone (1114-1187) sous le nom «Liber Karastonis », et a été commenté par de nombreux auteurs. Il existe quinze manuscrits du « Liber
Karastonis » qui ont été copiés entre le treizième et le seizième siècle, selon E. Moody et M. Clagett (voir [7]). Il comprend quatre théorèmes concernant la résolution du « problème de la poutre pesante ».

\section{2 «iber de Canonio»}

De même, il existe en latin un livre anonyme sous le nom : «Liber de Canonio » ou « Livre de la poutre pesante ». Ce livre a été édité par E. Moody en 1967, à partir de dix manuscrits copiés entre le treizième et le quatorzième siècle. Il comprend quatre théorèmes concernant la résolution du «problème de la poutre pesante » et quelques corollaires. P. Duhem (voir [29] a cru, ainsi que E. Moody, que le «Liber de Canonio» est une traduction directe du grec en latin d'un traité grec perdu. De même K. Jaouiche a prétendu qu'il a été écrit directement en latin au treizième siècle (voir [20], p. 73). Mais W. R. Knorr affirme (voir [30], p. 15) qu'il a comparé le « Liber de Canonio » écrit en latin avec « Ziāda fì al-Qarasțūn » ou «Appendice sur le qarasțūn » qui se trouve dans un manuscrit à Beyrouth et qu'il a trouvé que trois des théorèmes écrits dans ce dernier manuscrit sont identiques à la lettre avec une partie du « Liber de Canonio »; d'autres parties sont aussi superposables. Cela montre que le « Liber de Canonio a été traduit de l'arabe à partir d'un texte proche au moins du livre « Ziāda fī al-Qarastūn » qui est attribué à Thābit ibn Qurra (voir les détails de cette discussion dans [30], pp. 15-26).

\subsection{Excerptum de Libro Thebit de Ponderibus »}

Il existe un autre texte latin dont le titre comprend le nom de Thābit ibn Qurra : « Excerptum de Libro Thebit de Ponderibus » ou « passage du livre de Thābit sur la balance » que P. Duhem a signalé en 1905 dans son livre « Les origines de la statique $»$. Ce texte comprend treize propositions sur les poutres pesantes. Mais M. Clagett affirme (voir [7], p. 281, 1. 25-27) que les propositions contenues dans « Liber Karastonis » se trouvent dans leur totalité dans « Excerptum de Libro Thebit de Ponderibus » à l'exception d'une seule, mais dans l'ordre inverse.

\section{4 «iber Euclidis de Ponderoso et Levi »}

Il existe aussi un texte latin attribué d'après son titre à Euclide : « Liber Euclidis de Ponderoso et Levi Comparatione corporum and Invicem » ou « Le livre d'Euclide de la pesanteur et de la légèreté et la comparaison des corps les uns par rapport aux autres $»$. Ce texte traduit de l'arabe a été édité et traduit en anglais (voir [7], pp. 2131). Nous remarquons que le titre de ce texte ressemble à celui que nous avons cité plus haut et qui a été reproduit (et attribué à Euclide) par al-Khāzin̄̄ dans « Le livre de la balance de la sagesse $»$. Ce texte latin comprend neuf postulats et cinq théorèmes avec les démonstrations. On peut observer que le premier de ces théorèmes dit : « si des 
corps traversent des distances différentes dans des temps égaux, alors ceux qui traversent des distances plus grandes ont des forces plus grandes ». Ce théorème se base sur la théorie d'Aristote. Par ailleurs, E. Moody croit (voir [7], p. 24, 1. 42-43 et p. 25, 1. 1-5) que Thābit ibn Qurra s'est basé sur le résultat de ce théorème pour démontrer « La loi du levier» dans « Liber Karastonis ». Il croit aussi que le texte arabe qui a servi pour écrire ce texte latin a été écrit par Thābit en se basant sur un texte grec qu'il possédait au neuvième siècle (voir [7], p. 24, 1. 1-5).

\subsection{Des livres latins}

Certains traités écrits en latin au treizième siècle et aux siècles suivants ont emprunté de ces traductions latines des traités arabes. Nous en citons les traités suivants :

1. «Liber Archimedus de Insidentibus in Humidum » ou « Livre d'Archimède sur les corps flottants ». Il semble que l'auteur de ce livre connaissait les écrits latins traduits de l'arabe, puisque la deuxième partie de ce livre est une traduction latine d'une source arabe (voir [7], p. 38, 1. 44-46 et p. 39, 1. 1-5).

2. «Elementa super demonstrationem ponderum» ou «Les éléments sur la démonstration des poids », livre écrit par Jordanus de Nemore (Jordanus Nemorarius) qui est un mathématicien du treizième siècle. Ce traité a une relation évidente avec les écritures de Thābit ibn Qurra traduites en latin (voir [7], p. 123, 1. 32-39).

3. «Liber de Ponderibus » ou « Livre des poids » écrit par Jordanus de Nemore. Ce livre comprend une partie du livre précédent composé des sept postulats et des neuf théorèmes, tandis que les quatre théorèmes qui se trouvent à la fin de ce livre sont pris du « Liber de Canonio » cité plus haut (voir [7], p. 145, 1. 1-21).

4. «Liber de Ratione Ponderis» ou «Livre sur la théorie des poids » écrit aussi par Jordanus de Nemore. Il comprend quatre parties contenant 45 théorèmes avec leur démonstration. On y trouve aussi une partie des deux livres précédents. De même, il est influencé par les deux livres : «Liber Karastonis » et « Liber de Canonio » (voir les détails dans [7], pp. 169-173).

\section{Conclusion}

Nous avons essayé dans cet article de tracer les grandes lignes, connues jusqu'à maintenant, de l'histoire de la mécanique statique écrite en arabe. Nous avons ainsi passé en revue les sources grecques de la mécanique statique qui ont été traduites en arabe, comme les traités écrits par Aristote, Euclide, Archimède, Ménélaüs, Pappus, Philon de Byzance et Héron d'Alexandrie. Nous avons résumé, ensuite, les traités écrits en langue arabe de mécanique statique qui nous sont parvenus en entier : « Kitāb fī al-Qarasțūn » et « Ziāda fī al-Qarasțūn » de Thābit ibn Qurra et « le livre de la balance de la sagesse » de 'Abd al-Raḥmān al-Khāzin̄̄. Les autres traités arabes en mécanique statique sont considérés comme perdus jusqu'à maintenant, mais ils ont été cités par les biographes et par les savants qui ont utilisé leurs résultats et qui en ont reproduit des passages comme 'Abd Al-Raḥmān al-Khāzin̄̄ dans son livre.

Nous avons d'autre part cité des traités en mécanique statique écrits en latin, traduits de l'arabe ou inspirés par des traités écrits dans ce domaine en arabe.

Nous remarquons que c'est « le livre de la balance de la sagesse » d'al-Khāzin̄̄ qui joue le rôle principal dans l'écriture de l'histoire de la mécanique statique parvenue en langue arabe. Ce traité est, en effet, une véritable encyclopédie qui contient de nombreux passages des traités grecs qui ont été traduits en arabe et des traités qui ont été directement écrits en arabe dans le domaine de la mécanique statique. Un bon nombre de ces derniers (comme ceux de Abū Sahl al-Qūhī, al-Hassan ibn al-Haytham, Muzaffar al-Asfizārī, Muḥammad ibn Zakariyya al-Rāzī et 'Umar al-Khayyām) sont perdus et c'est grâce au livre d'al-Khāzin̄̄ que nous en avons des traces importantes.

Le livre d'al-Khāzin̄̄ qui a été écrit au début du treizième siècle nous donne ainsi une idée assez claire du niveau élevé des recherches entrepris en mécanique statique par les mathématiciens arabes qui ont utilisé les méthodes mathématiques développées à l'époque en algèbre et en géométrie.

Il reste devant les chercheurs en histoire des sciences beaucoup de travail à faire pour compléter et améliorer l'écriture de l'histoire de la mécanique statique écrite en arabe pendant la période médiévale.

\section{Références}

[1] Héron d'Alexandrie, Les Mécaniques, B. Carra de Vaux (Éd.), Introduction par D.R. Hill, Commentaires par A.G. Drachman, Les Belles Lettres, Paris, 1988

[2] Aristote, Mechanical Problems, English Translation, W.S. Hett, vol. 14, Cambridge, London, 1936, 1955, 1963, 1980

[3] Pappus, La Collection Mathématique, traduction française de Paul Ver Eecke, Paris, 1932-1982

[4] D.R. Hill, L'ingénierie civile et mécanique dans Encyclopédie de l'Histoire des Sciences Arabes, R. Rashed (Éd.), « Histoire des Sciences Arabes, vol. III, Paris, 1997, pp. 263-292

[5] Al-Khuwārizmī Abū Abd Allah Muḥammad ibn Aḥmad ibn Yūssef, Mafātīh al- 'ūlūm ou Les Clés des Sciences, Dār al-Maktaba al-'Ilmiyya, Beyrouth et aussi Le Caire, Idāra al-Ṭibā'a al-Munīriyya.

[6] Al-Khāzin̄̄ Abd al-Raḥmān, Kitāb Mīzān al-Ḥikma, ou Livre de la Balance de la Sagesse, Imprimerie du Cercle des Connaissances Ottomanes, Haydarābād, 1359 de l'Hégire (1941)

[7] E. Moody, M. Clagett, The Medieval Science of Weights, Madison, 1960

[8] Al-Qifțī, Tārikh al-Ḥukamā', J. Lippert (Éd.), Dieterich'sche Verlagsbuchhandlung, Leipzig, 1903

[9] F. Bancel, Histoire de la Statique Arabe, Mīzān al-Ḥikma d'al-Khāzin̄i, thèse, Université Paris 7, 2006 
[10] Ibn al-Nadīm Muammad ibn Isāq, Kitāb al-Fihrist, traduction anglaise par Barard Dodge: The Fihrist of alNadīm: A Tenth Century Survey of Muslim Culture, Columbia University Press, New York, 1970

[11] Banū Mūssā, Kitāb al-Ḥiyal ou Livre des Dispositifs Ingénieux, Ahmad Yūsef al-Ḥassan (Éd.), Université d'Alep, 1981

[12] Philon de Byzance, Le livre des appareils pneumatiques et des machines hydrauliques, traduction de Carra de Vaux (Éd.) dans Notices et Extraits des Manuscrits de la B. N., tome 38, Paris, 1903

[13] Al-Jazarī Abū al-'Izz ibn Ismā'īl al-Razzāz, Al-Jāmi‘a bayna al-'Ilm wa al-'Amal al-Nāfi'a fì Șinā'a al-Ḥiyal ou Le Manuel de la connaissance et de la pratique utile dans la fabrication des machines ingénieuses, Aḥmad Yūsef al-Ḥassan (Éd.), Université d'Alep, 1979

[14] F. Woepcke, Notice sur des traductions arabes de deux ouvrages perdus d'Euclide, J. Asiatique 4 (1851) 217-232

[15] Archimède, Les œuvres complètes, Traduction Paul Ver Eecke, Paris-Bruxelles, 1921

[16] T. Heath, The works of Archimede, Dover Publications, New York, pp. 1897-1912

[17] Archimède, Le Livre sur le Grave et le Léger, Zotenberg (Éd.), J. Asiatique 4 (1851) 217-232

[18] A.G. Drachman, Fragments from Archimede in Heron's Mechanics, Centaurus 8 (1963) 91-146

[19] E. Wiedmann, Die Schrift über den Qarastun, Bibliotheca Mathematica, Foldge III, XII (1912) $11-29$
[20] K. Jaouiche, Le Livre du Qarasțūn de Thābit ibn Qurra, Paris, 1976

[21] P. Abgrall, Le Développement de la Géométrie aux IX-XI siècles, Abū Sahl al-Qūhī, Blanchard, Paris, 2004

[22] R. Rashed, Les Mathématiques Infinitésimales, Tome I, London, 1993

[23] F. Bancel, Les Centres de Gravité d'Abū Sahl al-Qūhī, Arabic Sciences and Philosophy, 11 (2001) 45-78

[24] Ibn Abī Ușaybi'a, 'Uyūn al-Anbā' fì TTabaqāt al-Ațibbā' ou Les meilleures informations sur les catégories de médecins, Dār Maktaba al-Ḥayāt, Beyrouth, 1965

[25] Al-Qalqashandī Abū al-'Abbās Ahmad, Subh al-A'ashā ou L'aube du mal voyant, Le Caire, al-Mitba'a al-Amīriyya, 1913

[26] M.M. Rozenskaya, La Statique, dans R. Rashed (ed.), Histoire des Sciences Arabes 2 (1997) 263-292

[27] Al-B̄̄rūn̄i, Maqāla fī al-Nissab al-latī bayna al-Fillizāt Wa al-Jawāhir fì al-Hajm ou Traité sur les rapports entre les métaux et les pierres précieuses en volume, traduction russe par M.M. Rozenskaya, B.A. Rosenfeld, Nauchnoye nasledstvo 6 (1983) 141-162

[28] Al-B̄̄rūn̄i, Kitāb al-Jamāhir fì al-Jawāhir, Haydarābād, 1936, réimprimé à Beyrouth en 1984

[29] P. Duhem, Les Origines de la Statique, Paris, 1905

[30] W.R. Knorr, Ancient sources of the medieval tradition of the mechanics, Supplemento agli Annali dell'Instituto e Museo di Storia delle Scienza, Firenze, 1982 